\title{
Mitigation of optical fiber nonlinearities in OFDM systems using FWM-Aware Constellation Modulation Technique
}

\author{
C. Sánchez ${ }^{(1)}$, M. A. Z. A-Khateeb(1), Z. Tingting(1), F. Ferreira(1), P. Skvortcov(1), J. L. Wei(2), S. \\ Sygletos(1), A. D. Ellis ${ }^{(1)}$
}

(1) Aston Institute of Photonic Technologies (AIPT), Aston University, Birmingham, B4 7ET, UK c.sanchez-costa@aston.ac.uk

(2) Huawei Technologies Duesseldorf $\mathrm{GmbH}$, European Research Center, Riesstrasse 25, 80992 München, Germany.

Abstract A novel fiber nonlinearity mitigation technique is proposed where the constellation used for each subset of symbols is frame-wise optimized (rather than in average) to maximise resilience to the expected FWM allowing increased $\mathrm{GMI}$ and tolerable launch power ( $3 \mathrm{dBm})$.

\section{Introduction}

Optical orthogonal frequency division multiplexing (OFDM) and single carrier formats both suffer from nonlinearity in long haul transmission enhanced by the inherent or dispersion induced peak to average power ratio (PAPR). Previous attempts to combat this have relied on quasi static approaches such as digitalback-propagation, Volterra series equalization, probability and geometric shaping, perturbation techniques, or the nonlinear Fourier transform ${ }^{1}$ whose implementation is unaware of the transmitted bit sequence.

In this paper we introduce a four-wave mixing (FWM)-aware constellation modulation technique (FACT) to enhance the system tolerance to the accumulated nonlinear interference, explaining the principle and illustrating the benefit using OFDM. In FACT-assisted OFDM systems, the FWM effects for a given OFDM symbol are estimated at the transmitter side, and the constellation of a given subset of subcarriers are modified to make each OFDM symbol more robust to fiber nonlinearities. By applying constellation manipulation, FACT shares some features of geometric and probabilistic shaping and of coherent-WDM ${ }^{2}$, modifying constellation distributions and subcarrier phases to take into account the channel response respectively. However, FACT performs a frame-wise optimization rather than in average, making necessary to design the transceiver to accommodate this type of optimization.

\section{Description of FACT and simulated model}

The simulated system is shown in Fig.1(a). It consists of the basic modules of an OFDM system (blue modules following conventional designs ${ }^{3,4}$ ), while the FACT modules introduced here are shown in orange. Principle of FACT. At the transmitter, a constellation $C_{p}$ is selected from within $P$ available constellations. Each constellation consists of $M$ complex values, $S_{p}=$ $\left\{s_{p, 1}, s_{p, 2}, \ldots, s_{p, M}\right\}$ and has a message $(m)$-tosymbol mapping $\left(\Pi_{\mathrm{p}}(m)\right)$ associated, so the transmitted symbol on each subcarrier is given by:

$$
x_{k}=\Pi_{\mathrm{p}}\left(m_{k}\right), x_{k} \in S_{\mathrm{p}}, k \in\{1, N\}, \mathrm{p} \in\{1, P\}
$$

where $N$ is the number of transmitted subcarriers. Neglecting the memory effects thanks to the cyclic extension at the transmitter, we can express the $k$ th component after the fast Fourier transform (FFT) at the receiver as:

$y_{k}=x_{k}+i_{k}^{\prime}+n_{k}, k \in\{1, N\}$

where $i_{k}^{\prime}$ is the resulting FWM interference after chromatic dispersion compensation, and $n_{k}$ accounts for the noise. In selecting $C_{p}$ it is necessary to balance minimising the impact of FWM generated on other subcarriers, maximising the resilience to the expected FWM arising from other subcarriers, and enhancing the

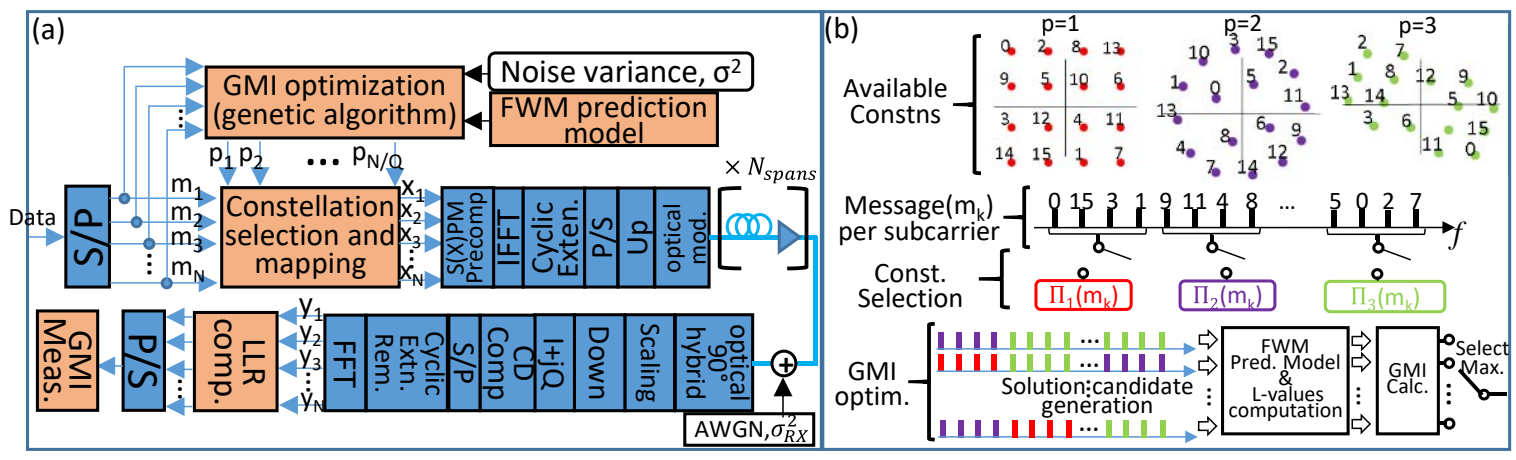

Fig. 1: (a) OFDM-FACT simulated model; (b) Principle of FACT 
anticipated linear noise tolerance. Constellation selection and mapping. To enable the receiver to estimate the constellations used, similarly to ${ }^{5}$, we divide the $N$ subcarriers into sets of size $Q$, such that all subcarriers in the same set use the same constellation. The $N$ symbols transmitted on each OFDM symbol can be expressed as the concatenation of vectors $\bar{x}_{q}=$ $\left[\Pi_{p_{q}}\left(m_{(q-1) Q+1}\right), \ldots, \Pi_{p_{q}}\left(m_{q Q}\right)\right], q \in\{1, N / Q\}$. Loglikelihood ratio (L-values) computation. Which message has been transmitted should in principle entail two decisions: firstly, to which constellation $y_{k}$ belongs to, and, secondly, which symbol within the chosen constellation has been transmitted. If these decisions are taken sequentially, an error in the constellation selection will increase substantially the number of wrongly decided bits. Instead, we compute a joint constellation/symbol log-likelihood ratio (L-value) as given in Eq. (3), for $N / Q$ sets (index q), $Q$ subcarriers within each set (index $\mathrm{k}$ ), and $\log _{2}(M)$ bits (index b). We extract information from the set of received symbols $\bar{y}_{q}$, rather than from a single received symbol. The first factor within the $p$ indexed sum is the conventional sum across the symbols within the $p$ th constellation with the bit in position $b$ equal to 1 or $0, S_{p, b}^{1}$ and $S_{p, b}^{0}$, respectively, while the second factor comes from the probability that the $p$ th constellation has been used, having received the set of symbols $\bar{y}_{q} \backslash\left\{y_{(q-1) Q+k}\right\}$. We call this factor interconstellation distance. Generalized mutual information optimization $(\mathrm{G} \overline{\mathrm{Ml}})$. We use a genetic algorithm $^{6}$ to maximise a metric based on the GMI, giving as result a set of constellation indices $\left\{p_{1}, \ldots, p_{N / Q}\right\}$ for each transmitted OFDM symbol independently. GMI is computed from the calculated L-values as in Eq.(30) of ${ }^{8}$. FWM prediction model. At the transmitter, in order to select the constellation, L-values are computed according to Eq. (3), using predicted values $\hat{y}_{k}=$ $x_{k}+\hat{\imath}_{k}^{\prime}$ and Gaussian noise. In order to calculate $\hat{\imath}_{k}^{\prime}$, we pre-compute all the FWM-triplets falling on the $k$ th subcarrier using the compressed Volterra series transfer function ${ }^{9}$. Together with the noise variance $\sigma^{2}$, this allows a prediction of the GMI to be made. Fig.1(b) shows an example of the process. We start (at the top of the figure) with 3 different 16-point constellations. 80 bits are transmitted on each OFDM symbol, and its partitioning in 20 messages of 4 bits to be transmitted on each subcarrier is shown in the second row. The 20 subcarriers are divided in sets of 4 . Messages to be transmitted on each subcarrier-set are mapped into complex symbols using one of the 3 constellations available (third row), resulting in $3^{5}=243$ possible constellation combinations. The search through the space of possible combinations is done by the genetic algorithm (bottom row), which computes the GMI from the L-value (Eq. (3)). Finally, the combination with highest GMI value is selected.

\section{Numerical Simulations}

We simulated a $1,000 \mathrm{~km}$ optical link with 10 spans of $100 \mathrm{~km}$ using the Split step method. The fiber model included second-order dispersion $(17 \mathrm{ps} /(\mathrm{km} \cdot \mathrm{nm}))$, Kerr nonlinearity $(1.3 /(\mathrm{W} \cdot \mathrm{km}))$, and loss $(0.2 \mathrm{~dB} / \mathrm{km})$. Amplification in each span was modelled by concatenating an ideal $20 \mathrm{~dB}$ gain amplifier with additive Gaussian white noise source giving a noise figure of $8 \mathrm{~dB}$. After the optical link, since the obtained GMI values for the conventional transmission were very close to the maximum achievable value, additional AWGN with a spectral density of $-119.89 \mathrm{dBm} / \mathrm{Hz}$ was added before optical detection. Intra and inter subcarrier nonlinear phase modulation effects were pre-compensated before the IFFT according to their theoretical expressions ${ }^{9}$. The FFT/IFFT size was 256, with 200 subcarriers used for data. The subcarrier spacing was $150.75 \mathrm{MHz}$ and a cyclic extension of $62.5 \%$ was used to enable dispersion tolerance. The signal was upsampled by a factor of 8 and scaled accordingly to the launch power; ideal linear field modulation and optical coherent detection were assumed. At the receiver, dispersion compensation was performed before OFDM demultiplexing into individual subcarrier via FFT and cyclic extension removal. We used 5 different 16-point constellations (see data set). The FWM predictor model was precomputed using 32-PSK and 100 OFDM training symbols. Simulations are carried out with either 10 or 20 subcarrier per set. The genetic algorithm used an initial population size of 100 , a generation gap of 0.99 , an elitist list of 1 individual, and a maximum number of generations equal to 40.Two different randomly generated binary streams were simulated, each one of 630 OFDM symbols. At the receiver, 624 OFDM symbols were processed and the GMI calculated over 100 different realizations of loaded receiver noise. The numerator and denominator in Eq. (3) were restricted to values higher than $10^{-100}$. GMI values were averaged over the 200 symbols transmitted per OFDM symbol, the 624 OFDM symbols, and the 100 loaded receiver noise and 2 signal realizations.

$$
L L R_{q k b}=\log \frac{\sum_{p=1}^{P}\left(\sum_{s \in S_{p, b}^{1}} \exp \left(-\frac{1}{\sigma^{2}}\left|y_{(q-1) Q+k^{\prime}}-s\right|^{2}\right) \cdot\left(\prod_{k^{\prime}=1, k^{\prime} \neq k}^{Q} \sum_{r=1}^{M} \exp \left(-\frac{1}{\sigma^{2}}\left|y_{(q-1) Q+k^{\prime}}-s_{p, r}\right|^{2}\right)\right)\right)}{\sum_{p=1}^{P}\left(\sum_{s \in S_{p, b}^{0}} \exp \left(-\frac{1}{\sigma^{2}}\left|y_{(q-1) Q+k}-s\right|^{2}\right) \cdot\left(\prod_{k^{\prime}=1, k^{\prime} \neq k}^{Q} \sum_{r=1}^{M} \exp \left(-\frac{1}{\sigma^{2}}\left|y_{(q-1) Q+k^{\prime}}-s_{p, r}\right|^{2}\right)\right)\right)}
$$




\section{Results}

Fig. 2 shows nonlinear threshold curves (expressed in GMI) for conventional OFDM transmission using Gray-coded 16QAM(blue); OFDM transmission using FACT with 10(green) and 20 (red) subcarriers per set, and the transmitter predicted GMI(black). Below 0dBm, conventional OFDM transmission outperforms

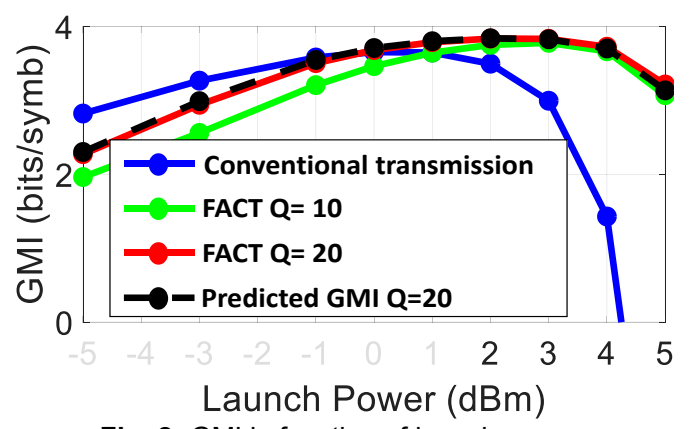

Fig. 2: GMI in function of launch power.

FACT. This is because even though the transmitter may have selected 16-QAM (one of the possible constellations) for all subcarriers and the noise tolerance should be indistinguishable, the receiver has no knowledge of which constellation has been used, penalizing in this way the system performance. This linear noise penalty is reduced as the system approaches closer to conventional transmission by increasing the number of subcarriers per set. Beyond $0 \mathrm{dBm}$, in the nonlinear regime, FACT clearly outperforms conventional transmission. More specifically, conventional transmission reaches a peak GMI value of 3.63 for a launch power of $0 \mathrm{dBm}$, while FACT reaches a peak value of 3.83 for a launch power of $3 \mathrm{dBm}$. This is because, the benefits of adjusting modulation format to mitigate nonlinearity outweighs linear SNR penalties of the different constellations and the lack of knowledge of the receiver on the constellation selection. The high accuracy of the FWM model used in this work is verified by the good matching between the estimated and received GMI. In Fig.3 we compare the distribution of $(-1)^{c_{q k b}} \cdot L L R_{q k b}\left(c_{q k b}\right.$ is the corresponding transmitted bit) for the conventional and FACT based OFDM systems for a launch power of $3 \mathrm{dBm}$. We can observe that the tail for positive values in the case of conventional transmission extends for longer, reflecting a penalty in the signal quality, which ultimately translates into a smaller GMI. A fourth curve in purple is also shown, where the interconstellation distance in Eq. (3) is not included. Its spreading to positive values illustrates its importance in the LLR calculation for FACT.

\section{Conclusions}

FACT, a novel fiber nonlinearity mitigation

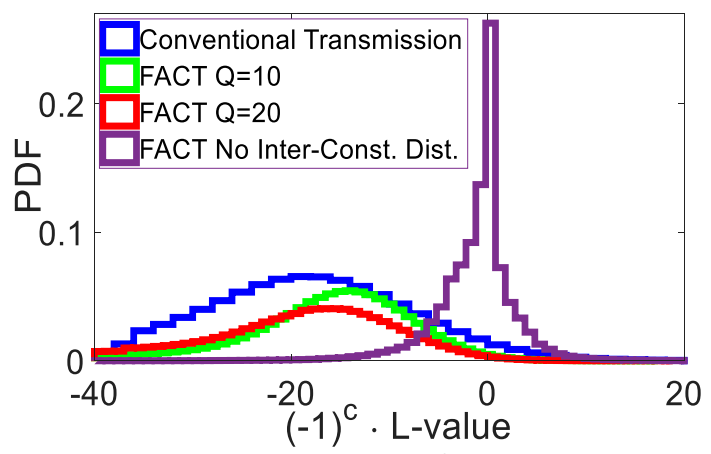

Fig. 3: Distribution of $(-1)^{c_{q k b}} \cdot L L R_{q k b}$

technique, has been presented in this work. Thanks to the estimation of the FWM effects on the signal, the frame-wise optimization and the detection based on L-values, FACT reaches higher achievable maximum rates and shows more robustness to fiber nonlinearities. Next steps include the extension of the work to higher order modulation formats, where higher improvements are expected.

\section{Acknowledgements}

The authors thank Gal Shulkind for his help. This work is funded by the EU projects INVENTION(659950), HSPACE(654809) and CEEOALAN(623515), and the EPSRC project PEACE(EP/L000091/1). https://doi.org/10.17036/researchdata.aston.ac.uk.00 $\underline{000347 .}$.

\section{References}

[1] J. C. Cartledge et al., "Digital signal processing for fiber nonlinearities," Opt. Express, Vol. 25, no. 3, p. 1916 (2017).

[2] F. C. G. Gunning et al., "Dispersion Tolerance of Coherent WDM", Photon. Technol. Lett., Vol. 18, no.12, p. 1338 (2006).

[3] W. Shieh and I. Djordjevic, OFDM for Optical Communications, Elsevier (2010).

[4] G. Agrawal, Fiber-Optic Communication Systems, Wiley (2011).

[5] S. S. Dash et al., "Constellation modulation - an approach to increase spectral efficiency," Opt. Express, Vol. 25, no. 14, p. 16310 (2017).

[6] A. J. Chipperfield, et al., "Genetic algorithm toolbox user's guide," Research Report 512, Dept. Autom. Control Syst. Eng., Univ. Sheffield, UK (1994).

[7] A. Alvarado, et al., "Replacing the soft-decision FEC limit paradigm in the design of optical communication systems," J. Lightw. Technol., Vol. 33, no. 20, p. 4338 (2015).

[8] G. Shulkind and M. Nazarathy, "Estimating the Volterra series transfer function over coherent optical OFDM for efficient monitoring of the fiber channel nonlinearity," Opt. Express, Vol. 20, no 27, p. 29035 (2012)

[9] M. Nazarathy et al., "Phased-array cancellation of nonlinear FWM in coherent OFDM dispersive multi-span links," Opt. Express, Vol. 16, no. 20, p. 15777 (2008). 